Original paper

\title{
NTCP modeling analysis of acute hematologic toxicity in whole pelvic radiation therapy for gynecologic malignancies - A dosimetric comparison of IMRT and spot-scanning proton therapy (SSPT)
}

\author{
Takaaki Yoshimura $^{\mathrm{a}, \mathrm{b}}$, Rumiko Kinoshita ${ }^{\mathrm{c}, *}$, Shunsuke Onodera ${ }^{\mathrm{b}, 1}$, Chie Toramatsu ${ }^{\mathrm{a}, 2}$, Ryusuke Suzuki ${ }^{\mathrm{d}}$, \\ Yoichi M. Ito ${ }^{\mathrm{e}}$, Seishin Takao ${ }^{a}$, Taeko Matsuura ${ }^{\mathrm{a}, \mathrm{f}, \mathrm{h}}$, Yuka Matsuzaki ${ }^{\mathrm{a}}$, Kikuo Umegaki ${ }^{\text {a,h }}$, Hiroki Shirato ${ }^{\text {b,f }}$, \\ Shinichi Shimizu f,g \\ a Proton Beam Therapy Center, Hokkaido University Hospital, Sapporo, Japan \\ ${ }^{\mathrm{b}}$ Department of Radiation Medicine, Graduate School of Medicine, Hokkaido University, Sapporo, Japan \\ ${ }^{\mathrm{c}}$ Department of Radiation Oncology, Hokkaido University Hospital, Sapporo, Japan \\ ${ }^{\mathrm{d}}$ Department of Medical Physics, Hokkaido University Hospital, Sapporo, Japan \\ e Department of Biostatistics, Hokkaido University Graduate School of Medicine, Sapporo, Japan \\ ${ }_{\mathrm{f}}$ Global Station for Quantum Medical Science and Engineering, Global Institution for Collaborative Research and Education (GI-CoRE), Hokkaido University, Sapporo, Japan \\ ${ }^{g}$ Department of Radiation Oncology, Graduate School of Medicine, Hokkaido University, Sapporo, Japan \\ ${ }^{\mathrm{h}}$ Division of Quantum Science and Engineering, Faculty of Engineering, Hokkaido University, Sapporo, Japan
}

\section{A R T I C L E I N F O}

Article history:

Received 12 April 2016

Received in Revised form 5 August 2016

Accepted 8 August 2016

Available online 25 August 2016

\section{Keywords:}

Whole pelvic radiation therapy

Spot-scanning proton therapy

LKB-NTCP model analysis

Treatment planning study

\begin{abstract}
A B S T R A C T
Purpose: This treatment planning study was conducted to determine whether spot scanning proton beam therapy (SSPT) reduces the risk of grade $\geqslant 3$ hematologic toxicity (HT3+) compared with intensity modulated radiation therapy (IMRT) for postoperative whole pelvic radiation therapy (WPRT).

Methods and materials: The normal tissue complication probability (NTCP) of the risk of HT3+ was used as an in silico surrogate marker in this analysis. IMRT and SSPT plans were created for 13 gynecologic malignancy patients who had received hysterectomies. The IMRT plans were generated using the 7-fields step and shoot technique. The SSPT plans were generated using anterior-posterior field with single field optimization. Using the relative biological effectives (RBE) value of 1.0 for IMRT and 1.1 for SSPT, the prescribed dose was $45 \mathrm{~Gy}(\mathrm{RBE})$ in $1.8 \mathrm{~Gy}(\mathrm{RBE})$ per fractions for $95 \%$ of the planning target volume (PTV). The homogeneity index (HI) and the conformity index (CI) of the PTV were also compared.

Results: The bone marrow (BM) and femoral head doses using SSPT were significantly lower than with IMRT. The NTCP modeling analysis showed that the risk of HT3+ using SSPT was significantly lower than with IMRT (NTCP $=0.04 \pm 0.01$ and $0.19 \pm 0.03, p=0.0002$, respectively). There were no significant differences in the $\mathrm{Cl}$ and $\mathrm{HI}$ of the PTV between IMRT and SSPT ( $\mathrm{CI}=0.97 \pm 0.01$ and $0.96 \pm 0.02, \mathrm{p}=0.3177$, and $\mathrm{HI}=1.24 \pm 0.11$ and $1.27 \pm 0.05, \mathrm{p}=0.8473$, respectively).

Conclusion: The SSPT achieves significant reductions in the dose to BM without compromising target coverage, compared with IMRT. The NTCP value for HT3+ in SSPT was significantly lower than in IMRT.

(c) 2016 Associazione Italiana di Fisica Medica. Published by Elsevier Ltd. This is an open access article under the CC BY-NC-ND license (http://creativecommons.org/licenses/by-nc-nd/4.0/).
\end{abstract}

\section{Introduction}

Whole pelvic radiation therapy (WPRT) plays an important role in the treatment of gynecologic cancers, especially cervical and

* Corresponding author at: Department of Radiation Oncology, Hokkaido University Hospital, N14W5 Kita-ku, Sapporo, Hokkaido 060-8638, Japan.

E-mail address: rumiko0220@pop.med.hokudai.ac.jp (R. Kinoshita).

1 Hokkaido Cancer Center, 2-3-54, Kikusui 4-jo, Shiroishi-ku, Sapporo, Hokkaido 003-0804, Japan

2 National Institute of Radiological Sciences, Research Center for Charged Particle Therapy, 4-9-1 Anagawa, Inage-ku, Chiba, Chiba 263-8555, Japan. endometrial cancers. Conventional WPRT contains approximately $40 \%$ of the total body bone marrow (BM) and results in hematologic toxicity (HT) [1]. Concurrent chemoradiotherapy (CCRT) is used to improve treatment outcome. However, adding chemotherapy increases the risk of severe HT. Severe HT is critical issue because it disturbs the execution of treatment on schedule such as interruptions of radiotherapy and holds or stops chemotherapy [2].

There are many studies that evaluate the relationship between the dosimetric parameter of BM and the severity of HT. Mell et al. have shown that a low dose irradiation volume of BM such as $\mathrm{V}_{10 \mathrm{~Gy}}$ 
and $\mathrm{V}_{20 \mathrm{~Gy}}$ has been associated with $\mathrm{HT}$ in chemoradiotherapy for cervical patients [1].

Recent advances in radiotherapy provide us with the superior conformal dose distribution, intensity modulated radiotherapy (IMRT) where photons enable a highly conformal dose distribution to targets. Several studies have demonstrated the utility of IMRT for WPRT. Dosimetric studies have shown that IMRT could reduce the irradiated volume of the BM compared with conventional treatment planning and clinical studies have suggested that IMRT reduced acute HT compared with conventional radiation therapy [3]. It has been proven that the reduction enabled by the low dose to the BM leads to reductions in the risk of severe HT [4].

Proton beam therapy (PBT) has a distinct physical characteristics known as the Bragg Peak, and PBT has been used to spare the normal tissue. Among several treatment delivery systems of PBT, the recent spot-scanning proton beam therapy (SSPT) system can provide a large treatment field $(30 \mathrm{~cm} \times 40 \mathrm{~cm})$ which can cover the whole pelvic region with one scanning field and enables delivery of a complex dose distribution $[5,6]$. Therefore, we hypothesized that SSPT could reduce the low dose to BM in WPRT compared with IMRT. A dosimetric comparison study has shown that intensity modulated proton beam therapy (IMPT) using SSPT was useful in sparing of pelvic BM, small bowel, rectum, and bladder compared with IMRT [7,8]. With IMPT it is possible to produce more complex dose distributions than SSPT with single field optimization (SFO), however IMPT is more susceptible to set up and range uncertainties than SFO $[9,10]$. When conducting clinical trials after an in silico study, it is preferable that SFO is selected as it is more robust than IMPT, and for this reason we decided to use SFO rather than IMPT. Moreover, SSPT using SFO has not been compared with IMRT yet. So, we do not know whether SSPT offers advantages compared to IMRT even without intensity modulation to reduce the dose to these organs. The first purpose of this study is to investigate whether SSPT using SFO reduces the dose to BM compared with IMRT for the organs at risk (OARs).

Also, to the best of our knowledge, the hypothesis of whether the reduction of the dose to BM by SSPT would result in risks of HT has not been evaluated in prospective clinical studies nor in simulation studies based on dosimetric results. Bazan et al. have suggested that the Lyman-Kutcher-Burman normal tissue complication probability (LKB-NTCP) based on dose volume statistics is useful as an in silico surrogate endpoint to estimate the risk of HT in patients who receive pelvic radiotherapy with chemotherapy $[11,12]$. To verify this, we have also investigated whether the reduction of the dose to BM by SSPT would result in the risk of HT comparing to IMRT using the LKB-NTCP model in this study.

The number of PBT facilities is increasing but the number of patients who can receive PBT is still limited. Therefore, it is necessary to be selective in determining who should benefit from PBT, before conducting clinical studies. The aim of this in silico study is to compare the dose of PBT to IMRT, and to evaluate the risk of adverse effects.

\section{Methods and materials}

\subsection{Patients}

This dosimetric study consists of 13 patients who had previously received radiotherapy to the pelvic region for adjuvant treatment or recurrent diseases in gynecological malignancies (cervical cancer; $n=8$, uterine body cancer; $n=4$, and ovarian cancer; $n=1$ ) at our institution from 2008 to 2014. All of them had received hysterectomy (with or without pelvic lymph node dissection). We created all plans anew based upon the virtual necessity of post-operative WPRT. This makes it possible to disregard the actual diagnoses or conditions of the patients. This study has been approved by the ethics committee of our hospital (014-0055).

\subsection{Contouring}

All patients received a planning computed tomography (CT) at a slice thickness of 2 or $2.5 \mathrm{~mm}$. The clinical target volume (CTV) was contoured on individual axial CT slices, according to the consensus guidelines for postoperative treatment of endometrial and cervical cancer [13]. The CTV included the common, external, and internal iliac lymph node regions, the upper vagina, parametrial and paravaginal soft tissue, and presacral lymph nodes. The planning target volume (PTV) was created by expanding the CTV with a $5 \mathrm{~mm}$ margin.

The bladder, rectum, bowel bag, femoral heads, and BM were contoured as the OARs according to the normal tissue contouring guidelines [14]. The bowel bag was contoured inferiorly above the rectum including both the small and large bowel and bowel loop, superiorly $2 \mathrm{~cm}$ above the PTV. The BM including the ilium, lower pelvis, and lumbosacral spine were contoured $2 \mathrm{~cm}$ superior/inferior to the PTV. All structures were contoured using the Pinnacle $^{3}$ treatment planning system (TPS) (ver.9.0; Philips, Inc., Madison, WI).

\subsection{Planning methods}

The IMRT plans were generated using Pinnacle ${ }^{3}$ TPS assuming photon treatment with an Clinac CL-iX (Varian Medical Systems, Palo Alto, CA) LINAC. We used seven evenly spaced intensity modulated fields which were generated with a 6 MV photon beam with a step and shoot multi leaf collimator technique.

The SSPT plans were calculated on the VQA TPS (Hitachi, Ltd., Hitachi, Japan), assuming proton treatment with a proton beam therapy system, PROBEAT-RT (Hitachi Co Ltd, Hitachi, Japan) [15]. Targets and normal structures were imported from Pinnacle ${ }^{3}$ TPS to VQA TPS via DICOM-RT. The SSPT plans consisted of an anterior-posterior (A-P) direction beam with the SFO method, in which each beam is optimized to deliver a uniform dose distribution to the target without intensity modulation [16], and not the multiple field optimization (MFO) method which is required for IMPT. There are several beam angles to choose for treatment of whole pelvic region in proton therapy. Lin et al. used the posterior oblique field technique [17] and Marnitz et al. used the three field technique (two oblique anterior beam and one posterior) [8]. We selected the AP-PA approach considering that we would be able to avoid differences in beam angles of individual patient which could affect the results.

Ninety-four energies between 70.2 and $220.0 \mathrm{MeV}$ are available for SSPT in our facility. The full width at half maximum (FWHM) of the spot size in air at the isocenter varies from $6.8 \mathrm{~mm}$ at $220.0 \mathrm{MeV}$ to $18.3 \mathrm{~mm}$ at $70.2 \mathrm{MeV}$, and the ellipticity of the beam is close to zero. The spot spacings in the horizontal and vertical directions are both determined automatically in the TPS, and distributed from 4.8 to $5.6 \mathrm{~mm}$ in this study.

The distal margin (DM), proximal margin (PM), and lateral margin (LM) were beam specific margins for the expansion from the CTV as an optimization volume for SSPT [16,18]. To account for the range uncertainty, the DM and PM were used as in the following equation,

$D M, P M=0.035 \times R+0.1 \mathrm{~cm}$

where $\mathrm{R}$ stands for the depth of the distal and proximal edges in water equivalent space and $0.1 \mathrm{~cm}$ is the direction of the proton delivery for the beam uncertainties [16]. The values of DM and PM for WPRT patients were $6.0-9.0 \mathrm{~mm}$ and $2.0-4.0 \mathrm{~mm}$, respectively. The target volume must be expanded laterally from the 
Table 1

Summary of the results of the DVHs analysis for the PTV and organs at risk.

\begin{tabular}{|c|c|c|c|c|c|}
\hline Organ & Parameter & Objectives & IMRT & SSPT & $P$ value \\
\hline \multirow[t]{5}{*}{ PTV } & $\mathrm{D}_{93 \%}[\%]$ & $>99$ [\%] & $99.91 \pm 0.15$ & $100.00 \pm 0.01$ & 0.0352 \\
\hline & $\mathrm{D}_{95 \%}[\%]$ & $>95[\%]$ & $95.17 \pm 0.20$ & $95.25 \pm 0.21$ & 0.3101 \\
\hline & $\mathrm{D}_{110 \%}[\%]$ & $<10[\%]$ & $0.39 \pm 1.01$ & $0.66 \pm 0.76$ & 0.1855 \\
\hline & CI & & $0.97 \pm 0.01$ & $0.96 \pm 0.02$ & 0.3177 \\
\hline & HI & & $1.24 \pm 0.11$ & $1.27 \pm 0.05$ & 0.8473 \\
\hline \multirow[t]{2}{*}{ Bone marrow } & $\mathrm{V}_{10 G y}[\%]$ & $<90$ [\%] & $83.47 \pm 2.30$ & $55.14 \pm 3.00$ & 0.0002 \\
\hline & $\mathrm{V}_{\text {20Gy }}[\%]$ & $<75[\%]$ & $64.86 \pm 3.18$ & $42.63 \pm 5.20$ & 0.0002 \\
\hline \multirow[t]{2}{*}{ Rectum } & Max dose [cGy] & & $4756.43 \pm 107.80$ & $4749.34 \pm 101.54$ & 0.4730 \\
\hline & $\mathrm{V}_{40 \mathrm{~Gy}}[\%]$ & $<60[\%]$ & $53.34 \pm 5.79$ & $50.68 \pm 15.28$ & 0.1219 \\
\hline \multirow[t]{2}{*}{ Bladder } & Max dose [cGy] & & $4768.18 \pm 105.55$ & $4845.83 \pm 93.84$ & 0.0133 \\
\hline & $\mathrm{V}_{45 \mathrm{~Gy}}[\%]$ & $<35$ [\%] & $23.35 \pm 11.01$ & $26.16 \pm 13.13$ & 0.1367 \\
\hline \multirow[t]{2}{*}{ Bowel bag } & Max dose [cGy] & & $4875.67 \pm 112.70$ & $4965.82 \pm 81.47$ & 0.0009 \\
\hline & $\mathrm{V}_{40 G y}[\%]$ & $<30[\%]$ & $25.81 \pm 4.19$ & $24.61 \pm 4.92$ & 0.1082 \\
\hline Femoral heads & $\mathrm{V}_{30 \mathrm{~Gy}}[\%]$ & $<15[\%]$ & $7.28 \pm 4.53$ & $2.09 \pm 2.34$ & 0.0002 \\
\hline
\end{tabular}

CTV to ensure appropriate dose coverage of the target volume considering internal target motion and setup errors [18]. With this, the LM including the internal margin and set-up margin can be calculated by a summation of these two factors as follows,

$L M=0.04 \times \mathrm{S}+0.3 \mathrm{~cm}$

where $\mathrm{S}$ is the maximum size of the CTV, as uncertainties in target localization are considered to increase with increases in the target size. The values of LM for WPRT patients were $8.5-11.5 \mathrm{~mm}$.

In this article, the dose unit of Gray (Gy) represents the dose weighted by the relative biological effectives (RBE). An RBE value of 1.0 was employed for the photon IMRT and 1.1 for SSPT plans.

The prescribed dose was $45 \mathrm{~Gy}$ in 25 fractions. All plans were designed so that $95 \%$ of the PTV received the prescription dose, at least $99 \%$ of the PTV received $93 \%$ of the prescribed dose and no more than $10 \%$ of the PTV received $110 \%$ of the prescribed dose.

The dose constrains for the BM were defined as $V_{10 G y}<90 \%$ and $\mathrm{V}_{20 \mathrm{~Gy}}<75 \%$, as previous studies have shown that $\mathrm{V}_{10 \mathrm{~Gy}}$ or $\mathrm{V}_{20 \mathrm{~Gy}}$ is associated with severe HT [1]. For other OARs, the following constrains were used: rectum $\mathrm{V}_{40 G y}<60 \%$, bladder $\mathrm{V}_{45 \mathrm{~Gy}}<35 \%$, bowel bag $\mathrm{V}_{40 \mathrm{~Gy}}<30 \%$, femoral heads $\mathrm{V}_{30 \mathrm{~Gy}}<15 \%$ and the maximum dose to the rectum, bladders and bowel bag should not exceed $50 \mathrm{~Gy}$, the dose constraints for the PTV and OARs are summarized in Table 1 [19]. A plan was considered acceptable when it met at least the prescribed dose to the PTV and the maximum dose to the OARs.

\subsection{Evaluation}

The dose volume histograms (DVHs) were generated for the PTV and all OARs. The $\mathrm{V}_{10 \mathrm{~Gy}}$ and $\mathrm{V}_{20 \mathrm{~Gy}}$ of the BM were calculated and compared to evaluate the risk of severe HT. The different levels of irradiated volumes of other OARs: $\mathrm{V}_{45 \mathrm{~Gy}}$ of the bladder, $\mathrm{V}_{40 \mathrm{~Gy}}$ of the rectum and bowel bag, and $V_{30 G y}$ of the femoral heads were also calculated and evaluated.

To compare target conformity and heterogeneity, the conformity index (CI) and homogeneity index (HI) of PTV were calculated for the IMRT and SSPT plans. There are various definitions of $\mathrm{CI}$ and $\mathrm{HI}$ in previous studies [20], and here we used the following formulas: $C I=V_{p} / V_{\text {PTV }}$ where $V_{p}$ is the target volume enclosed by the prescribed dose line, and $V_{\text {PTV }}$ is the volume of the PTV; $\mathrm{HI}=\mathrm{D}_{\max } / \mathrm{D}_{\min }$ where $\mathrm{D}_{\min }$ and $\mathrm{D}_{\max }$ were the minimum and maximum doses of the PTV. These analyses were performed with non-shifted plans without modification of the CT values, namely those of the nominal plan.

Two different approaches were employed in the evaluation of the robustness of the target coverage and the representative OARs. The uncertainties related to patient positioning were evaluated by six different plans with a $\pm 5 \mathrm{~mm}$ (left-right (L-R), anterior- posterior (A-P) and superior-inferior (S-I)) isocenter shift on the planning CT (shifted plan) [17,21]. The effect of interfractional anatomical changes such as emptying or filling of air cavities was evaluated by a plan termed HU (HU plan), generated by recalculating with the Hounsfield Units of the bowel bag as $20 \mathrm{HU}$, which was the mean value of several areas neighboring the bowel tissue in the bowel bag. The DVHs were generated for CTV and the OARs in all shifted and HU plans to evaluate the robustness.

\subsection{Lyman-Kutcher-Burman-normal tissue complication probability (LKB-NTCP) model and gEUD}

As the in silico surrogate for the risk of HT we used the LKB-NTCP model [22-24] with Bazan's methods [11,12]. The LKB-NTCP model is used to estimate the complication probabilities for nonuniformaly irradiated tissue based on DVHs. The Lyman-NTCP [22] describes the probability of complications for a uniform dose, $\mathrm{D}$, to the organ as

$$
\begin{aligned}
& N T C P=\varphi(t)=\frac{1}{\sqrt{2 \pi}} \int_{-\infty}^{t} \exp \left(-\frac{x^{2}}{2}\right) d x \\
& t=\frac{D-T D_{50}}{m \cdot T D_{50}} \\
& T D_{50}(v)=T D_{50}(1) \cdot v^{-n}
\end{aligned}
$$$$
v=\frac{V}{V_{\text {ref }}}
$$

These equations have 4 parameters, $\mathrm{TD}_{50}, \mathrm{~m}, \mathrm{n}$, and $\mathrm{V}_{\text {ref. }}$ The $\mathrm{TD}_{50}$ value is the tolerance dose for a $50 \%$ complication probability for uniform doses to the organ; $\mathrm{m}$ is a dimensionless parameter to determine the slope of the complication probability according to dose curve, and $\mathrm{n}$ is the parameter for the volume dependence of the complication probability, finally $\mathrm{V}_{\text {ref }}$ is a reference volume for the OARs [25]. $\mathrm{TD}_{50}(1)$ is the tolerance dose of the whole organ irradiation.

For the uniform dose D in Eq. (4), we used the generalized equivalent uniform dose (gEUD) [26,27]. Here, the gEUD was calculated as

$g E U D=\left(\sum_{i=1}^{k} v_{i} d_{i}^{a}\right)^{1 / a}$

where a is a parameter equal to $1 / n$, which parameter $n$ is the volume dependence of the complication probability [25], and k represents unequal fractional sub-volumes. The $d_{i}$ term is the dose for each bin in a differential DVHs, and $v_{i}$ is the volume in a specific 


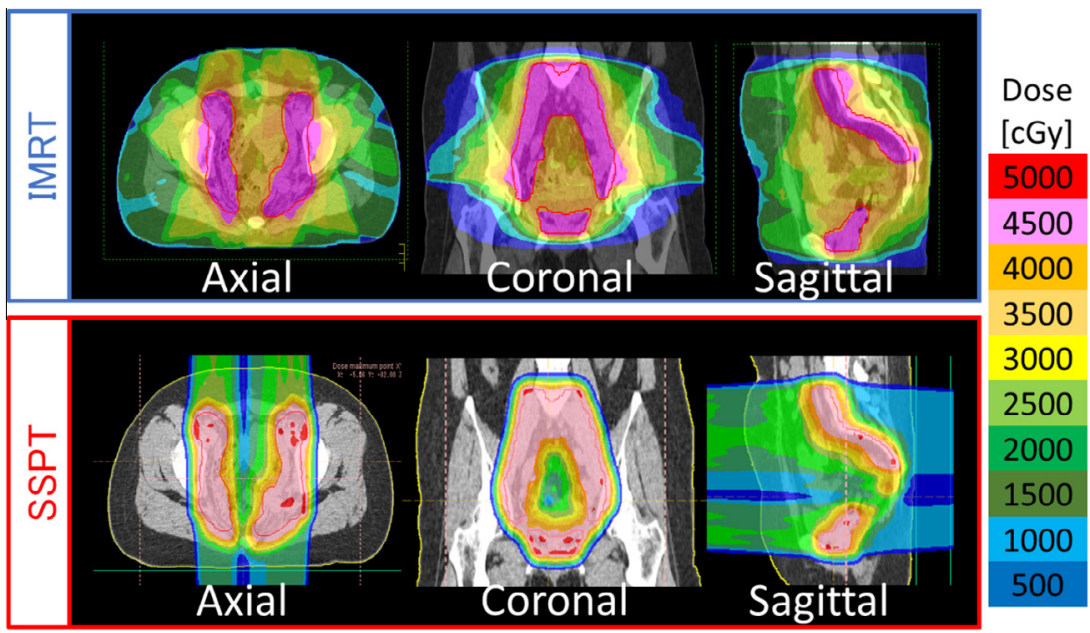

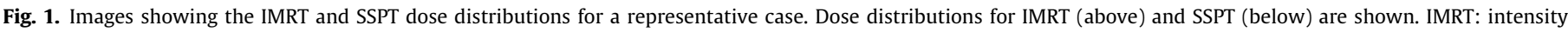
modulated radiation therapy, SSPT: spot scanning proton therapy.

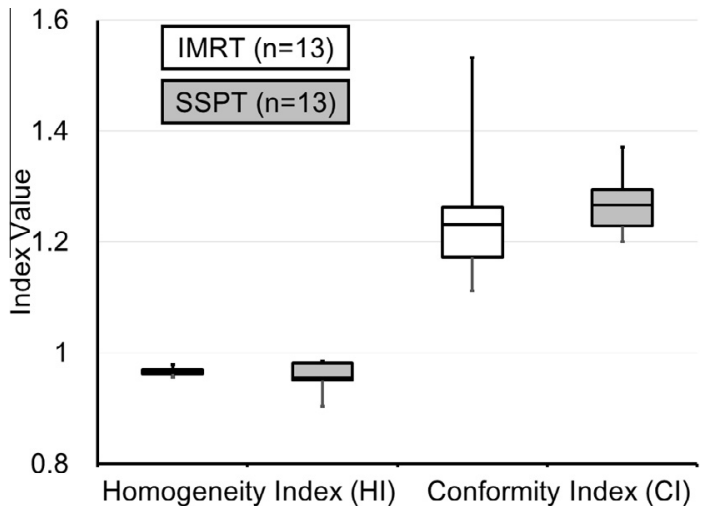

Fig. 2. Boxplots of the IMRT (white) and SSPT (gray) of CI and HI for the PTV. IMRT: intensity modulated radiation therapy, SSPT: spot scanning proton therapy, PTV: planning target volume, $\mathrm{CI}$ : conformity index, $\mathrm{HI}$ : homogeneity index.

dose bin $i$. The OARs irradiated with uniform gEUD resulted in the same NTCP as the original inhomogeneous dose distribution.

Bazan et al. analyzed the relationship between dose volume data and the incidence of HT in 32 patients receiving CCRT for gynecological malignancies [12]. There they applied the LKBNTCP model for the dose-response curve of HT equal to or higher than Grade3 (HT3+) according to the Common Terminology Criteria for Adverse Events ver.4.0 [28] and found the parameters $n=1$, $\mathrm{m}=0.27$, and $\mathrm{TD}_{50}=35 \mathrm{~Gy}$ in Eqs. (4) and (5) [12].

\subsection{Statistical analysis}

The $\mathrm{CI}$ and the HI of the PTV, DVHs of the PTV and each OARs, gEUD for BM, and NTCP value for HT3+ were compared for the IMRT and SSPT. The Wilcoxon signed rank test was used for all statistical comparisons. A $p$ value of $<0.05$ was considered statistically significant. All statistical analyses were performed by JMP Pro 11 (SAS Institute Inc., Cary, NC, USA).

\section{Results}

\subsection{DVHs analysis}

Twenty-six plans were generated (13 IMRT plans and 13 SSPT plans), with plan comparisons shown in Fig. 1. All plans achieved

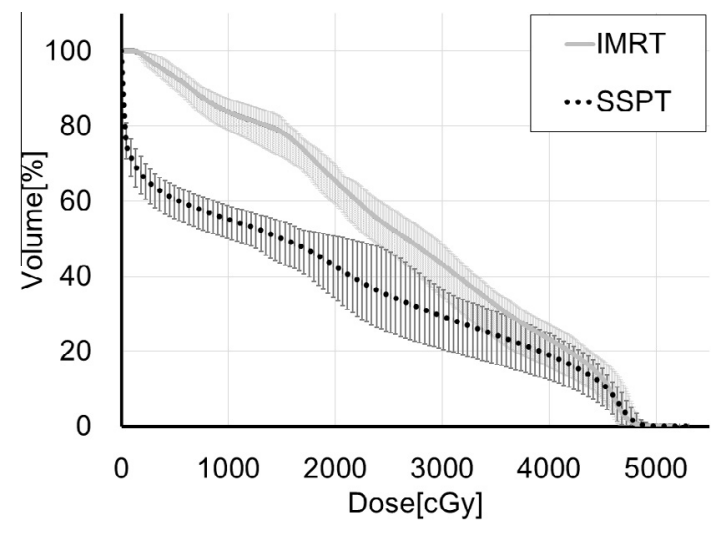

Fig. 3. Dose volume histograms (DVHs) for the bone marrow (BM). The solid and dot line represents the average dose volume histogram of the 13 patients for IMRT (line) and SSPT (dot line), respectively, while the surrounding shading represents the range for the 13 patients. In the SSPT plan, we were able to reduce the low dose region of the BM below that of the IMRT plan.

the prescribed dose to the PTV maintaining doses for the OARs below their respective dose constrains.

There were no statistically significant differences in $\mathrm{CI}$ or $\mathrm{HI}$ of PTV between the IMRT and SSPT plans $(\mathrm{CI}=0.97 \pm 0.01$ for IMRT and $0.96 \pm 0.02$ for SSPT, $\mathrm{HI}=1.24 \pm 0.11$ for IMRT and $1.27 \pm 0.05$ for SSPT and $\mathrm{p}=0.3177,0.8473$, respectively) (Fig. 2).

The volumes of BM receiving 10 and 20 Gy using SSPT were significantly lower than with IMRT $\left(\mathrm{V}_{10 \mathrm{~Gy}}=83.47 \pm 2.30 \%\right.$ for IMRT and $55.14 \pm 3.00 \%$ for SSPT, $V_{20 G y}=64.86 \pm 3.18 \%$ for IMRT and $42.63 \pm 5.20 \%$ for SSPT and $\mathrm{p}=0.0002,0.0002$, respectively). Fig. 3 plots the DVHs data for BM, showing that the SSPT demonstrated lower irradiated volumes below $30 \mathrm{~Gy}$ for BM.

The SSPT also decreased the $\mathrm{V}_{30 \mathrm{~Gy}}$ of the femoral heads $(7.28 \pm 4.53 \%$ for IMRT and $2.09 \pm 2.34 \%$ for SSPT, and $\mathrm{p}=0.0002)$. On the other hand, there were no significant differences between IMRT and SSPT for the $\mathrm{V}_{45 \mathrm{~Gy}}$ of the bladder, and the $\mathrm{V}_{40 \mathrm{~Gy}}$ of the rectum and bowel bag. The DVHs for the femoral heads, rectum, bladder, and bowel bag are shown in Fig. 4. Table 1 shows a detailed summary of DVHs results for the PTV and the OARs.

The results of the evaluation of the robustness for target coverage are detailed in Table 2. For the CTV D98, the dose received by $98 \%$ of the volume of the CTV, was measured in each shifted plan. Changes in the mean value of CTV D98 of the shifted plans for the 13 patients were within $1 \%$ in both IMRT and SSPT (left: $-0.6 \%$ for 
(a) Femoral heads

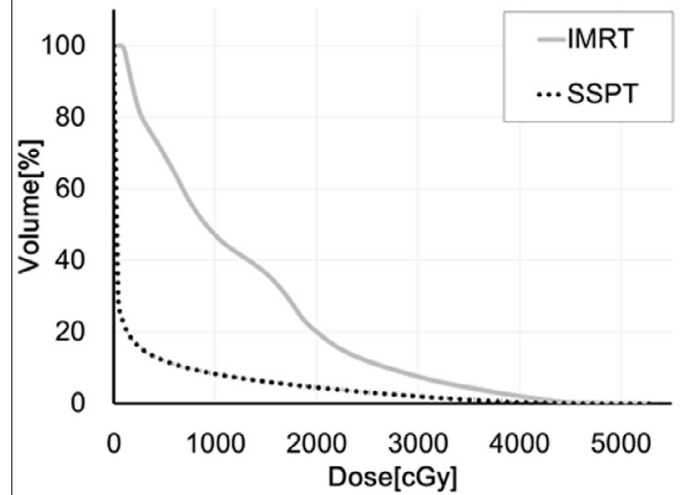

(c) Rectum

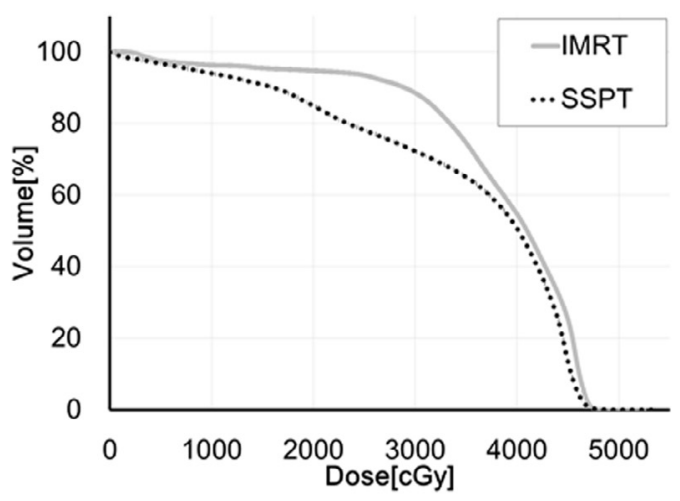

(b) Bowel bag

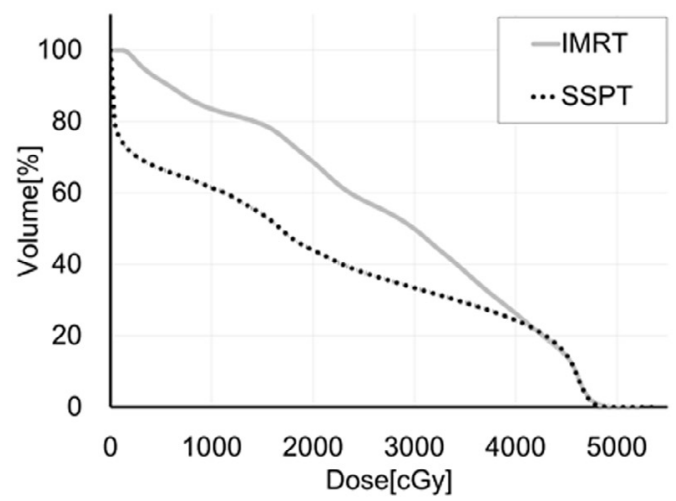

(d) Bladder

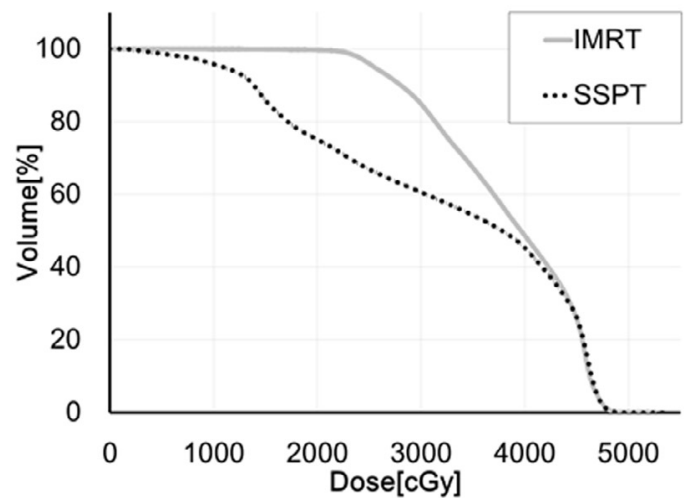

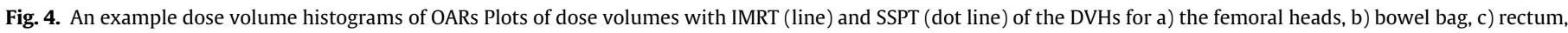
and d) bladder.

Table 2

Mean and standard deviations of CTV D98 in the 13 patients for the nominal plan, the HU plan, and the plans shifted $5 \mathrm{~mm}$ along each axis.

\begin{tabular}{lll}
\hline & IMRT [cGy] & SSPT [cGy] \\
\hline Nominal & $4580.77 \pm 36.62$ & $4517.75 \pm 69.06$ \\
HU & $4544.62 \pm 38.43$ & $4472.14 \pm 205.98$ \\
Left & $4551.54 \pm 24.44$ & $4496.69 \pm 44.85$ \\
Right & $4555.38 \pm 20.66$ & $4520.36 \pm 67.49$ \\
Anterior & $4546.92 \pm 40.90$ & $4514.16 \pm 70.77$ \\
Posterior & $4539.23 \pm 32.78$ & $4493.40 \pm 48.66$ \\
Superior & $4543.08 \pm 52.66$ & $4489.11 \pm 55.78$ \\
Inferior & $4554.62 \pm 38.65$ & $4499.21 \pm 53.57$ \\
\hline
\end{tabular}

Abbreviations: CTV D98: the dose received by $98 \%$ of the volume of the CTV, Nominal: nominal plan, HU: plan in which the value of the Hounsfield Units in the bowel bag was replaced by $20 \mathrm{HU}$, Left: plan shifted $5 \mathrm{~mm}$ to the left, Right: plan shifted $5 \mathrm{~mm}$ to the right, Anterior: plan shifted $5 \mathrm{~mm}$ to the anterior, Posterior: plan shifted $5 \mathrm{~mm}$ to the posterior, Superior: plan shifted $5 \mathrm{~mm}$ to the superior, Inferior: plan shifted $5 \mathrm{~mm}$ to the inferior directions respectively.

IMRT and $-0.5 \%$ for SSPT, right: $-0.6 \%$ and $-0.5 \%$, anterior: $-0.7 \%$ and $0.1 \%$, posterior: $-0.9 \%$ and $-0.1 \%$, superior: $-0.8 \%$ and $-0.6 \%$, inferior: $-0.6 \%$ and $-0.4 \%$, respectively). The changes in the mean values of CTV D98 of HU plans were $-0.8 \%$ in IMRT and $-1.0 \%$ in SSPT. The largest change of HU plan reached $-14.3 \%$ in SSPT, whereas the changes were within $3 \%$ in all IMRT plans.

We evaluated the robustness for the representative OARs, BM, and femoral heads, in which significant differences between IMRT and SSPT were observed in the dose volume analysis using the nominal plan as mentioned above; the $V_{10 G y}$ and $V_{20 G y}$ of BM, and $V_{30 G y}$ of femoral heads were calculated in each shifted plan.

The changes in the mean value of BM $V_{10 G y}$ and $V_{20 G y}$ of the shifted plans for the 13 patients were within $1 \%$ in both IMRT and SSPT ( $\mathrm{V}_{10 \mathrm{~Gy}}$; left: $0.0 \%$ for IMRT and $-0.6 \%$ for SSPT, right: $-0.1 \%$ and $-0.2 \%$, anterior: $0.7 \%$ and $-0.2 \%$, posterior: $-0.7 \%$ and $0.2 \%$, superior: $-1.0 \%$ and $-0.8 \%$, inferior: $0.7 \%$ and $0.1 \%$. $\mathrm{V}_{20 \mathrm{~Gy}}$; left: $-0.4 \%$ and $-0.3 \%$, right: $0.0 \%$ and $-0.2 \%$, anterior: $-0.4 \%$ and $0.2 \%$, posterior: $0.0 \%$ and $-0.2 \%$, superior: $-0.8 \%$ and $0.0 \%$, inferior: $0.5 \%$ and $-0.8 \%$, respectively). The changes in the mean values of $V_{10 G y}$ and $\mathrm{V}_{20 \mathrm{~Gy}}$ for the BM of HU plans were within $3 \%\left(\mathrm{~V}_{10 \mathrm{~Gy}} ; 0.0 \%\right.$ for IMRT and $-0.3 \%$ for SSPT. $\mathrm{V}_{20 \mathrm{~Gy}} ;-0.3 \%$ and $-2.3 \%$, respectively). In one case the changes in the $\mathrm{V}_{20 \mathrm{~Gy}}$ of the HU plan was $-11.7 \%$ in SSPT. Fig. 5 plots the DVHs data for the BM of the plan robustness (Fig. 5(a) for the shifted plans and Fig. 5(b) for the HU plans), which show that the SSPT demonstrated a lower irradiated volume below $30 \mathrm{~Gy}$ as with the nominal plan (shown in Fig. 3).

The changes in the mean value of the femoral head $V_{30 G y}$ of the shifted plans for the 13 patients were from $-39.6 \%$ to $59.5 \%$ in IMRT and from $-78.1 \%$ to $380.9 \%$ in SSPT. The changes in the mean value $\mathrm{V}_{30 \mathrm{~Gy}}$ for the femoral heads of HU plans were within $0.5 \%$ $\left(-0.1 \%\right.$ for IMRT and $-0.3 \%$ for SSPT). All femoral heads $\mathrm{V}_{30 \mathrm{~Gy}}$ of the shifted plans and the HU plans were below $9 \%$ in SSPT. The $\mathrm{V}_{30 \mathrm{~Gy}}$ was exceeded $15 \%$ in two cases of IMRT. The $\mathrm{V}_{30 \mathrm{~Gy}}$ of SSPT was significantly smaller than with IMRT in both the shifted and HU plans ( $p=$ left: 0.0002 , right: 0.0002 , anterior: 0.0002 , posterior: 0.0002 , superior: 0.0002 , inferior: 0.0005 and $\mathrm{HU}$ : 0.0002 , respectively).

\subsection{Estimated risk of $\mathrm{HT} 3+$}

The NTCP values for HT3+ for the IMRT and SSPT plans were $0.19 \pm 0.03$ and $0.04 \pm 0.01$, respectively, with the SSPT results showing statistically significantly lower mean NTCP values than with IMRT ( $p=0.0002$ ). The gEUD of BM for IMRT and SSPT were 
(a) Shift

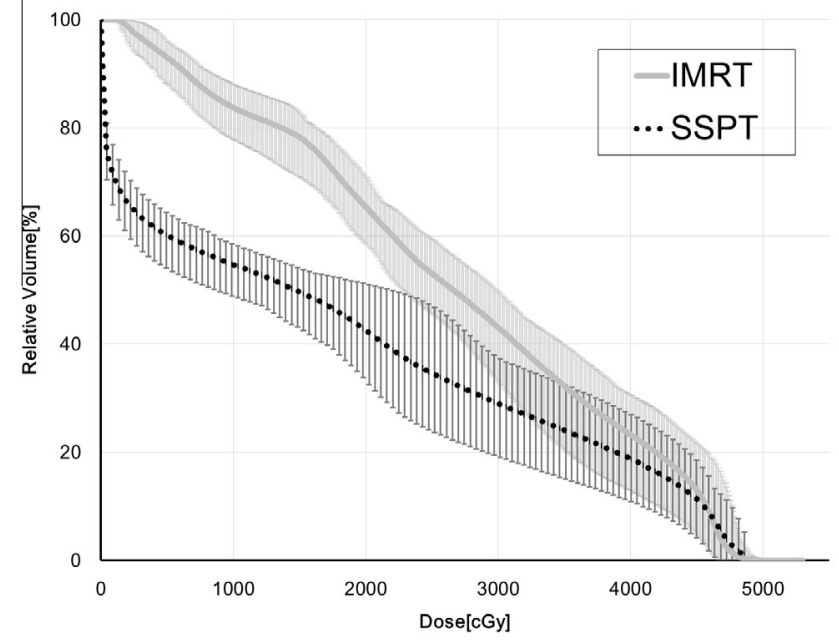

(b) $\mathrm{HU}$

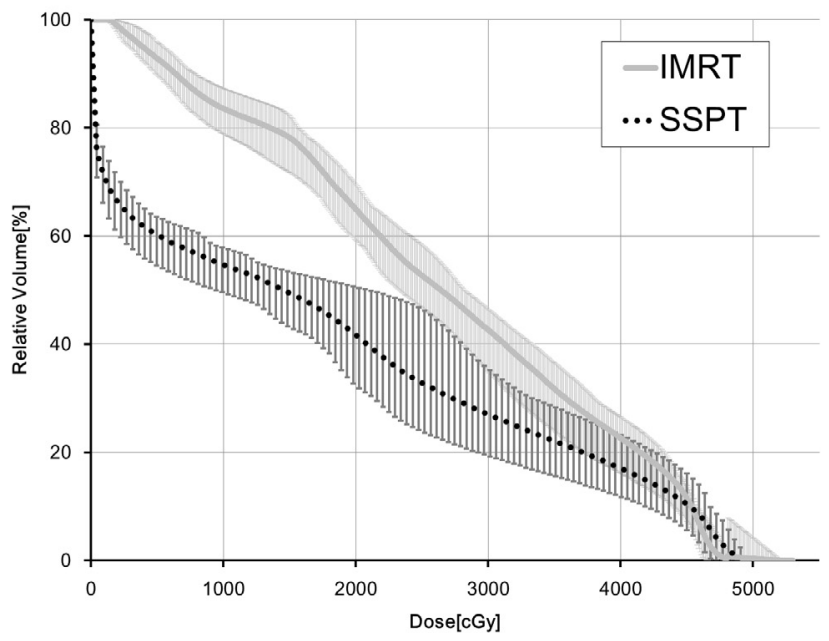

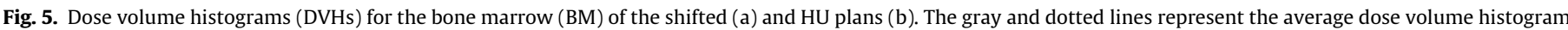
of the 13 patients with IMRT and SSPT, respectively for shifted (a) and HU plans (b). The surrounding shading represents the range for the 13 patients.

$2663.32 \pm 101.91 \mathrm{cGy}$ and $1793.32 \pm 154.60 \mathrm{cGy}$, respectively, and the SSPT was statistically significantly below the IMRT gEUD of $\mathrm{BM}(\mathrm{p}=0.0002)$.

We plotted the NTCP value versus gEUD of the BM for the IMRT and SSPT according to the Bazan method (Fig. 6) [12]. The predicted NTCP for HT3+ was below 0.05 under $1950 \mathrm{cGy}, 0.10$ at 2290 cGy, and doubled to 0.20 at 2710 cGy. This shows that the predicted NTCP value gradually increases above around 2000 cGy. The maximum gEUD for SSPT was 2020 cGy with an NTCP value of 0.059 . The maximum and minimum gEUD for IMRT were 2663 cGy and 2402 cGy, and the NTCP values here were 0.21 and 0.12 .

\section{Discussion}

The main objective of this treatment planning study is to investigate if the risk of HT is reduced using SSPT with SFO compared to photon IMRT. Previously, there have been a small number of clinical reports of PBT for the treatment of gynecological malignancies $[17,29]$. The small number of studies is related to the fact that it was difficult to generate a large treatment field by passive scattering PBT systems which had been widely used so far. The present study showed that SSPT is able to cover the whole of the pelvic region with one scanning field $(30 \mathrm{~cm} \times 40 \mathrm{~cm})$ and that this is useful to reduce the risk of HT when compared with IMRT. In addition, since the large scanning field makes the irradiation time longer, scan path optimization is one of the way for cost-effective treatment delivery [30].

Our results show that SSPT reduced the low dose irradiation volumes of BM such as the $\mathrm{V}_{10 \mathrm{~Gy}}$ and $\mathrm{V}_{20 \mathrm{~Gy}}$. Dinges et al. showed that IMPT can spare BM compared to IMRT at a wide range of dose levels, and our results are consistent with those results [7]. However, a difference in the dose volume statistics itself does not necessarily affect the risk of HT in clinical outcomes. Using the LKB-NTCP model and parameters in our assumptions, the present study shows that the NTCP value for HT3+ in SSPT, even without intensity modulation, was significantly lower than in IMRT. This indicates that SSPT has a positive impact on clinical outcomes regarding HT when comparing with IMRT.

Although randomized studies are needed to confirm if PBT reduces the toxicity comparing to IMRT, it is hard to conduct such studies. To estimate and evaluate the effectiveness of PBT over

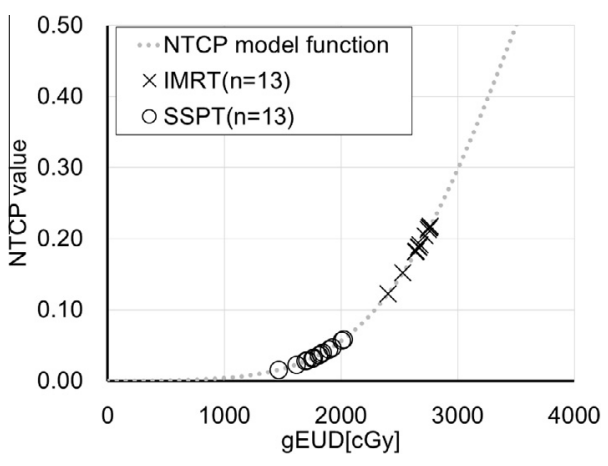

Fig. 6. Layman-Kucher-Burman normal tissue complication probability (LKB-NTCP) model for grade 3 hematologic toxicity (HT3+) A plot of NTCP values for IMRT $(x)$ and SSPT $(O)$ with the generalized equivalent uniform dose (gEUD). With the SSPT plan, it was possible to reduce both of the values of NTCP and gEUD below those of the IMRT plan.

photon therapy, several studies using the NTCP modeling analysis exist. Jakobi et al. conducted in silico study using NTCP modeling analysis in head and neck region to identify patients who may benefit from PBT [31-33]. Makishima et al. showed that NTCP value for the lung and heart decreased in proton plan compared with photon plan in esophageal cancer [34]. Toramatsu et al. showed that large size of hepatocellular carcinoma could be more safely treated with SSPT than IMRT regarding the risk of radiation-induced liver disease using NTCP modeling analysis [35]. To the best of our knowledge, our study is the first study to evaluate the risk of HT by NTCP modeling analysis using PBT.

We also compared the DVHs data of other OARs. Here the SSPT decreased the dose to the femoral heads compared to IMRT (statistically significantly), but there were no significant differences in the doses to the bowel bag, the bladder, or rectum. There are two possible reasons why our results are different from previous studies. Marnitz et al. showed that, compared to IMRT, IMPT using three fields (two oblique anterior beams and one posterior) reduced the dose to the bowel bag and rectum significantly [8]. Here we used AP-PA fields with SFO, and differences in the beam angle and optimization methods could have affected the results. More work is required to conclude whether the dose reduction could result in lower NTCP with these OARs. 
Although, as the current situation, treatment uncertainty such as set-up error, range uncertainty, imaging uncertainty, and others is the issue to be solved in IMPT $[9,10]$. To consider these uncertainties, robustness optimization was being developed and it was available only a limited TPS [36]. Since TPS and quality assurance procedures are progressively improving, MFO would be the sensible choice in the WPRT.

We confirmed that the $\mathrm{CI}$ and $\mathrm{HI}$ for the PTV were comparable in SSPT and IMRT in the study here. These results indicate that SSPT can achieve reductions in doses to the BM without sacrificing the dose coverage of the PTV. Marnitz et al. have shown that the CI and $\mathrm{HI}$ for the target were similar in a dosimetric study to compare IMRT, helical tomotherapy, volumetric arc therapy (VMAT), and IMPT for twenty patients suffering from cervical cancer [8]. Our results are consistent with the Marnitz results.

One critical issue of SSPT is the effect of uncertainties related to the set-up and interfractional anatomical changes. Differences of D98 for CTV between the nominal and shifted plans were within $1 \%$ in SSPT. However, there was a large difference, more than $10 \%$, between the nominal and HU plan in one case in SSPT. This may indicate that the robustness of SSPT is inferior to IMRT in some cases.

The average changes of $\mathrm{V}_{20 \mathrm{~Gy}}$ for the $\mathrm{BM}$ of the $\mathrm{HU}$ plan were $-2.3 \%$ in SSPT, and in one case the change in $\mathrm{V}_{20 \mathrm{~Gy}}$ of the HU plan was $-11.7 \%$. All values of the $\mathrm{V}_{20 \mathrm{~Gy}}$ and $\mathrm{V}_{10 \mathrm{~Gy}}$ for the BM of the HU plans were smaller than nominal, so the risk of HT would not be larger than the nominal value.

Large changes were seen in $\mathrm{V}_{30 \mathrm{~Gy}}$ for the femoral heads of the shifted plans. The reason for this phenomenon is owing to the very small value of $\mathrm{V}_{30 \mathrm{~Gy}}$ for the femoral head in SSPT. In one case, $\mathrm{V}_{30 \mathrm{~Gy}}$ was $0.02 \%$ in the nominal plan, and $0.31 \%$ in the shifted plan, so the change was larger than $1500 \%$. The $\mathrm{V}_{30 \mathrm{~Gy}}$ of SSPT was significantly smaller than IMRT in both shifted and HU plans, so the results of the dose to the femoral heads would not be changed by the effects of treatment uncertainties.

A shortcoming of this study is that we have not evaluated the functional part of the BM in the pelvic bone and assumed the whole of the pelvic bone as active BM. Dinges et al. have used nuclear imaging, (18)F-fluorothymidine positron emission tomography, to specify the functional BM area in the pelvic bone [7]. The differences observed in this study may be somewhat altered if we had used functional imaging, we feel that the difference would not be large however.

Another limitation of this study is that we conducted the evaluation of the effects of anatomical changes by replacing the Hounsfield Unit of the bowel bag, not by using several CT scans taken during the course of the treatment to evaluate the effects of interfractional uncertainties due to bowel gas and anatomical changes [37].

The study is also limited by possible biases in the assumptions of the study. Among these the usefulness of NTCP and gEUD is still debated. Also, the parameters proposed by Basan et al. have not yet been validated in prospective clinical studies. The present study suggests that SSPT could offer a new way ahead for WPRT combined with chemotherapy, although whether SSPT actually does reduce the incidence and/or severity of HT has to be confirmed in clinical studies.

\section{Conclusions}

Compared with IMRT, SSPT using SFO can achieve significant reductions in the dose to $\mathrm{BM}$ with adequate dose coverage to the PTV. The LKB-NTCP value for the HT3+ of SSPT is significantly smaller than that of IMRT. These results indicate the advantage of WPRT with SSPT using SFO considering the risk of severe HT and possibly to the femoral heads. However, for dose reductions to the bowel, rectal tissue, and bladder adverse effects, SSPT using SFO is inadequate.

\section{Acknowledgements}

A preliminary version of this study was presented at the 57th Annual Meeting of the American Society of Radiation Oncology (ASTRO), 2015.10.18-21, San Antonio. This work was supported by JSPS KAKENHI Grant numbers 15K19760, 25461899, 15H04899, $15 \mathrm{H} 04768$.

\section{References}

[1] Mell LK, Kochanski JD, Roeske JC, Haslam JJ, Mehta N, Yamada SD, et al. Dosimetric predictors of acute hematologic toxicity in cervical cancer patients treated with concurrent cisplatin and intensity-modulated pelvic radiotherapy. Int J Radiat Oncol Biol Phys 2006;66:1356-65.

[2] Albuquerque K, Giangreco D, Morrison C, Siddiqui M, Sinacore J, Potkul R, et al. Radiation-related predictors of hematologic toxicity after concurrent chemoradiation for cervical cancer and implications for bone marrowsparing pelvic IMRT. Int J Radiat Oncol Biol Phys 2011;79:1043-7.

[3] Mell LK, Tiryaki H, Ahn KH, Mundt AJ, Roeske JC, Aydogan B. Dosimetric comparison of bone marrow-sparing intensity-modulated radiotherapy versus conventional techniques for treatment of cervical cancer. Int J Radiat Oncol Biol Phys 2008;71:1504-10.

[4] Brixey CJ, Roeske JC, Lujan AE, Yamada SD, Rotmensch J, Mundt AJ. Impact of intensity-modulated radiotherapy on acute hematologic toxicity in women with gynecologic malignancies. Int J Radiat Oncol Biol Phys 2002;54:1388-96.

[5] Knopf AC, Lomax A. In vivo proton range verification: a review. Phys Med Biol 2013;58:R131-60.

[6] Shimizu S, Matsuura T, Umezawa M, Hiramoto K, Miyamoto N, Umegaki K, et al. Preliminary analysis for integration of spot-scanning proton beam therapy and real-time imaging and gating. Phys Med 2014;30:555-8.

[7] Dinges E, Felderman N, McGuire S, Gross B, Bhatia S, Mott S, et al. Bone marrow sparing in intensity modulated proton therapy for cervical cancer: efficacy and robustness under range and setup uncertainties. Radiother Oncol 2015;115:373-8.

[8] Marnitz S, Wlodarczyk W, Neumann O, Koehler C, Weihrauch M, Budach V, et al. Which technique for radiation is most beneficial for patients with locally advanced cervical cancer? Intensity modulated proton therapy versus intensity modulated photon treatment, helical tomotherapy and volumetric arc therapy for primary radiation - an intraindividual comparison. Radiat Oncol 2015;10:91.

[9] Lomax AJ. Intensity modulated proton therapy and its sensitivity to treatment uncertainties 1: the potential effects of calculational uncertainties. Phys Med Biol 2008;53:1027-42.

[10] Lomax AJ. Intensity modulated proton therapy and its sensitivity to treatment uncertainties 2: the potential effects of inter-fraction and inter-field motions. Phys Med Biol 2008;53:1043-56.

[11] Bazan JG, Luxton G, Mok EC, Koong AC, Chang DT. Normal tissue complication probability modeling of acute hematologic toxicity in patients treated with intensity-modulated radiation therapy for squamous cell carcinoma of the anal canal. Int J Radiat Oncol Biol Phys 2012;84:700-6.

[12] Bazan JG, Luxton G, Kozak MM, Anderson EM, Hancock SL, Kapp DS, et al. Impact of chemotherapy on normal tissue complication probability models of acute hematologic toxicity in patients receiving pelvic intensity modulated radiation therapy. Int J Radiat Oncol Biol Phys 2013;87:983-91.

[13] Small Jr W, Mell LK, Anderson P, Creutzberg C, De Los SantosJ, Gaffney D, et al. Consensus guidelines for delineation of clinical target volume for intensitymodulated pelvic radiotherapy in postoperative treatment of endometrial and cervical cancer. Int J Radiat Oncol Biol Phys 2008;71:428-34.

[14] Gay HA, Barthold HJ, O’Meara E, Bosch WR, El Naqa I, Al-Lozi R, et al. Pelvic normal tissue contouring guidelines for radiation therapy: a Radiation Therapy Oncology Group consensus panel atlas. Int J Radiat Oncol Biol Phys 2012;83: e353-62.

[15] Umezawa M, Fujimoto R, Umekawa T, Fujii Y, Takayanagi T, Ebina F, et al. Development of the compact proton beam therapy system dedicated to spot scanning with real-time tumor-tracking technology. AIP Conf Proc 2013;1525:360-3.

[16] Zhu XR, Poenisch F, Song X, Johnson JL, Ciangaru G, Taylor MB, et al. Patientspecific quality assurance for prostate cancer patients receiving spot scanning proton therapy using single-field uniform dose. Int J Radiat Oncol Biol Phys 2011:81:552-9.

[17] Lin LL, Kirk M, Scholey J, Taku N, Kiely JB, White B, et al. Initial report of pencil beam scanning proton therapy for post hysterectomy patients with gynecologic cancer. Int J Radiat Oncol Biol Phys 2015;95:181-9.

[18] Park PC, Zhu XR, Lee AK, Sahoo N, Melancon AD, Zhang L, et al. A beam-specific planning target volume (PTV) design for proton therapy to account for setup and range uncertainties. Int J Radiat Oncol Biol Phys 2012;82:e329-36. 
[19] Radiation Therapy Oncology Group (RTOG). 0921 <https://www.rtog.org/ ClinicalTrials/ProtocolTable/StudyDetails.aspx?study=0921/>; [accessed 2015.11.11].

[20] Feuvret L, Noel G, Mazeron JJ, Bey P. Conformity index: a review. Int J Radiat Oncol Biol Phys 2006;64:333-42.

[21] Sio TT, Merrell KW, Beltran CJ, Ashman JB, Hoeft KA, Miller RC, et al. Spotscanned pancreatic stereotactic body proton therapy: a dosimetric feasibility and robustness study. Phys Med 2016;32:331-42.

[22] Lyman JT. Complication probability as assessed from dose-volume histograms. Radiat Res Suppl 1985;8:S13-9.

[23] Kutcher GJ, Burman C. Calculation of complication probability factors for nonuniform normal tissue irradiation: the effective volume method. Int J Radiat Oncol Biol Phys 1989;16:1623-30.

[24] Kutcher GJ, Burman C, Brewster L, Goitein M, Mohan R. Histogram reduction method for calculating complication probabilities for three-dimensional treatment planning evaluations. Int J Radiat Oncol Biol Phys 1991;21:137-46.

[25] Burman C, Kutcher GJ, Emami B, Goitein M. Fitting of normal tissue tolerance data to analytic function. Int J Radiat Oncol Biol Phys 1991;21:123-35.

[26] Luxton G, Keall PJ, King CR. A new formula for normal tissue complication probability (NTCP) as a function of equivalent uniform dose (EUD). Phys Med Biol 2008;53:23-36.

[27] Niemierko A. A generalized concept of equivalent uniform dose (EUD). Med Phys 1999;26:1100 (Abstract).

[28] Common Terminology Criteria for Adverse Events V4.0 (CTCAE). <http://ctep. cancer.gov/protocolDevelopment/electronic_applications/ctc.htm\#ctc_40/>; 2009 [accessed 2015.09.16].

[29] Yanazume S, Arimura T, Kobayashi H, Douchi T. Potential proton beam therapy for recurrent endometrial cancer in the vagina. J Obstet Gynaecol Res 2015;41:813-6.
[30] Dias MF, Riboldi M, Seco J, Castelhano I, Pella A, Mirandola A, et al. Scan path optimization with/without clustering for active beam delivery in charged particle therapy. Phys Med 2015;31:130-6.

[31] Jakobi A, Bandurska-Luque A, Stutzer K, Haase R, Lock S, Wack LJ, et al. Identification of patient benefit from proton therapy for advanced head and neck cancer patients based on individual and subgroup normal tissue complication probability analysis. Int $\mathrm{J}$ Radiat Oncol Biol Phys 2015;92:1165-74.

[32] Jakobi A, Luhr A, Stutzer K, Bandurska-Luque A, Lock S, Krause M, et al. Increase in tumor control and normal tissue complication probabilities in advanced head-and-neck cancer for dose-escalated intensity-modulated photon and proton therapy. Front Oncol 2015;5:256.

[33] Jakobi A, Stutzer K, Bandurska-Luque A, Lock S, Haase R, Wack LJ, et al. NTCP reduction for advanced head and neck cancer patients using proton therapy for complete or sequential boost treatment versus photon therapy. Acta Oncol 2015:54:1658-64.

[34] Makishima H, Ishikawa H, Terunuma T, Hashimoto T, Yamanashi K, Sekiguch $\mathrm{T}$, et al. Comparison of adverse effects of proton and X-ray chemoradiotherapy for esophageal cancer using an adaptive dose-volume histogram analysis. J Radiat Res 2015:56:568-76.

[35] Toramatsu C, Katoh N, Shimizu S, Nihongi H, Matsuura T, Takao S, et al. What is the appropriate size criterion for proton radiotherapy for hepatocellula carcinoma? A dosimetric comparison of spot-scanning proton therapy versus intensity-modulated radiation therapy. Radiat Oncol 2013;8:48.

[36] Liu W, Zhang X, Li Y, Mohan R. Robust optimization of intensity modulated proton therapy. Med Phys 2012;39:1079-91.

[37] Blanco Kiely JP, White BM. Robust proton pencil beam scanning treatment planning for rectal cancer radiation therapy. Int J Radiat Oncol Biol Phys 2016;95:208-15. 